\title{
肝脏增强 CT 与肝脏 MRI 在诊断肝癌中的作用对比
}

\section{Comparison of the Role of Liver Enhanced CT and Liver MRI in the Diagnosis of Hepatocellular Carcinoma \\ 罗瑞华 赵荣娟 于联芳}

Ruihua Luo Rongjuan Zhao Lianfang Yu

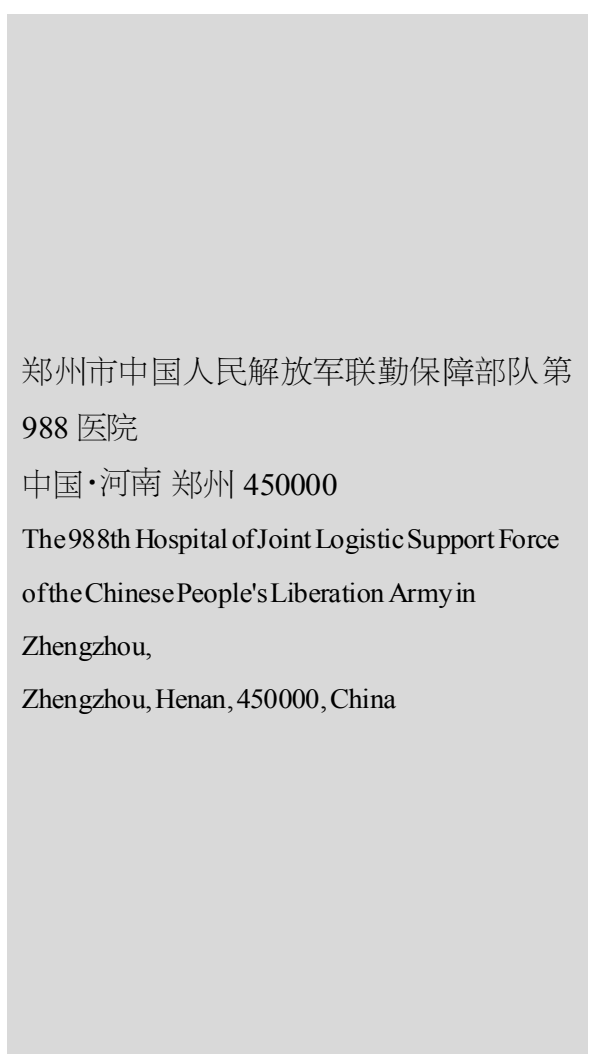

\section{1 引言}

在整个消化系统当中，原发性肝癌为其最常见的一种恶 性肿瘤, 在各类肿瘤当中, 仅次于肺癌、胃癌之后, 居于第 3 位, 有着比较高的发病与死亡率, 但需要指出的是, 大多患者 一经发现即为中晚期, 且其预后与早期沴断之间, 有着十分紧 密的关系 ${ }^{[1]}$ 。所以, 采取切实措施, 提高肝癌的早期诊断准确 率, 对于患者预后改善有着积极意义。现阶段, 临床当中比较 常用的检查方法有 MRI、CT 等, 且实施动态增强检查, 有助于 肝局灶性病变检出率的提升, 但哪种最为突出, 有待明确。本 文针对所收治的原发性肝癌患者, 分别实施肝脏增强 CT 与 MRI 检查, 对比其准确率, 现对此做了深究。

\section{2 资料与方法}

\section{1 一般资料}

2018 年 3 月一 2019 年 3 月, 选取来本院接受治疗的原发
性肝癌患者 50 例, 均与中国抗癌协会肝癌专业委员会最新修 订的诊断标准相符 ${ }^{[2]}$, 且手术开始前均病理检查确诊, 其中 30 例患者经手术确诊, 20 例患者经皮肝穿刺活检而确诊。诸如肝 区疼痛、上腹饱胀、乏力纳差及消瘦等为其主要临床表现。其 中, 男性 28 例, 女 22 例, 最小年龄 30 岁, 最大 70 岁, 中位 $(51.4 \pm 4.2)$ 岁; 最短病程 $15 \mathrm{~d}$, 最长 12 个月, 中位 $(4.2 \pm 0.5)$ 个 月。肿瘤部位: 13 例左边半肝, 31 例右边半肝, 6 例左右半肝。

\section{2 方法}

(1)CT 检查。用 Light Speed 64 排螺旋扫描仪 (GE, 128 层), 电流为 $150 \mathrm{~mA}$, 电压为 $120 \mathrm{kV}$, 层厚/层间隔比值为 $10 \mathrm{~mm}$ 。用 20 100mL 非离子造影剂碘海醇, $300 \mathrm{mgI} / \mathrm{mL}$, 以 $3 \sim$ $4 \mathrm{~mL} / \mathrm{s}$ 的流率, 以高压注射方式从肘静脉给药, 开展动态增强 扫描, 分别在延迟期( 180s )、静脉期 (60s)、动脉期 (25s)开展扫 描操作。(2)MRI 扫描。利用 ESSENZA1.5T 超导磁共振成像仪 (西门子), 开展 T1WI、T2WI 加权扫描, 层厚控制在 $5 \mathrm{~mm}$, 层间 
隔为 $1 \sim 2 \mathrm{~mm}$ 。将钝喷替酸葡甲胺 (GdDTPA) 作为对比剂, 经肘 静脉高压注射, 开展增强扫描, 控制流速, 即 $1.5 \sim 2.0 \mathrm{~mL} / \mathrm{s}$, 用量 控制在 $0.1 \mathrm{mmol} / \mathrm{kg}_{0}$ 。扫描期同 CT 检查。

\section{3 统计学处理}

SPSS20.0 处理数据, $t / \chi^{2}$ 检验, $P<0.05$ 表示差异有统计学 意义。

\section{3 结果}

\subsection{CT 增强扫描表现}

(1)动脉期。病灶出现显著强化 45 例, 其密度较周围正常 肝组织, 显著偏高, 呈等密度强化 3 例, 没有明显强化 2 例。(2) 门脉期。病灶呈现为等密度 48 例, 呈现为略高密度 2 例。(3)延 迟期。在具体的强化程度上有一定下降,病灶都呈现为略低或 者低密度。

\section{$3.2 \mathrm{MRI}$ 的表现}

经平扫得知, 在 T1WI 上, 呈现为低或者略低信号, 而在具 体的 T2WI 上,显示略高或者不均匀高信号 47 例, 显示等信号 3 例。通过增强扫描得知, 动脉期得到显著强化, 且呈现为高信 号, 较周围肝组织明显偏高。门脉期病灶等信号 45 例, 低信号 5 例; 而在延长期, 低或者略低信号 49 例, 1 例等信号。

\section{3 各检查方法的诊断结果对比}

经增强 CR 检查, 共检出肝内病灶 39 例, 肝外病灶 18 例; 而经 MRI 检查, 共检出肝内病灶 47 例, 肝外病灶 28 例。MRI 检出肝内外病灶阳性率相比增强 CT, 都显著偏高 $(P<0.05)$, 如 表 1 所示。

表 1 各检查方法的诊断结果对比 [例 (\%) ]

\begin{tabular}{c|c|c|c}
\hline 方法 & 例数(例) & 肝外病灶阳性率 $(\%)$ & 肝内病灶阳性率 $(\%)$ \\
\hline CT & 50 & $39(78.00)$ & $18(36.00)$ \\
\hline MRI & 50 & $47(94.00)$ & $28(56.00)$ \\
\hline$\chi^{2}$ & & 4.56 & 3.48 \\
\hline$P$ 值 & & $<0.05$ & $<0.05$ \\
\hline
\end{tabular}

\section{4 对不同肿瘤大小的诊断结果对比}

在所选取患者中, 肝脏增强 CT 检出肝癌 39 例, MRI47 例, MRI 诊断准确率较 CT, 显著偏高 $(P<0.05)$ 。针对直径 $\leqslant 3 \mathrm{~cm}$ 肿瘤, MRI 诊断准确率较 CT 偏高 $(P<0.05)$, 而针对直径大于 $3 \mathrm{~cm}$ 肿瘤, 两者比较, 无显著差异 $(P>0.05)$, 如表 2 所示。

表 2 对不同肿瘤大小的诊断结果对比 [例 (\%)]

\begin{tabular}{c|c|c|c}
\hline 方法 & $>3 \mathrm{~cm}(19$ 例 $)$ & $\leqslant 3 \mathrm{~cm}(31$ 例 $)$ & 合计 \\
\hline CT & $17(89.47)$ & $22(70.97)$ & $39(78.00)$ \\
\hline MRI & $18(94.74)$ & $29(93.55)$ & $47(94.00)$ \\
\hline$\chi^{2}$ & 0.10 & 5.24 & 5.13 \\
\hline$P$ 值 & $<0.05$ & $>0.05$ & $<0.05$ \\
\hline
\end{tabular}

\section{4 讨论}

在中国各类型恶性肿瘤当中, 原发性肝癌发病率位居第 3 位,仅次于肺癌、胃癌,且以乙型肝炎表面抗原阳性、丙型肝炎 病毒、乙型肝炎病毒者最易发生。据相关数据统计得知, 直 径 $\leqslant 3.0 \mathrm{~cm}$ 的小肝癌, 其五年生存率为 $65 \sim 75 \%$ 向 , 而中晚期者 的生存率仅为 $30 \%$ 左右。近年, 伴随当今医疗技术, 尤其是影 像学技术的不断提升与完善, 诸如 MRI、CT 等已经成为检查、 诊断原发性肝癌的重要手段。需要说明的是,肝脏增强 CT 表 现无论与肿瘤血供之间,还是与肝动脉血供间,均有着紧密关 联。许多肝癌的供血由肝动脉来实现, 因此, 在动脉期,开展快 速且准确的扫描, 门静脉血还没有达到肝脏, 因此, 肝癌肿块 会呈现为显著强化; 如果处于门脉期, 就难以持续强化, 其密 度会出现快速降低, 突出表现为快显快出, 但需要指出的是, 其在诊断低供血肿瘤上,具有局限性。而针对 MRI 扫描技术而 言, 其具有诸多优点, 如有着比较高的软组织分辨率、图像采 集信号多以及没有放射性辐射等。从本文结果得知, MRI 平扫 $\mathrm{T} 1 \mathrm{WI}$ 上显示为略低或者低信号, 而在 T2WI 上, 显示略高或者 高信号的有 47 例, 即有典型的癌样信号。研究结果得知, MRI 肝内、肝外病灶阳性率高于增强 $\mathrm{CT}_{\text {。 }}$ 针对有明显块影或者是 有转移灶、病灶局限的肝内胆管细胞癌, 增强 MRI、增强 CT 能 够比较快速且准确地做出诊断，而针对呈散在分布、沿着胆管 浸润生长的肝外病灶, 二者均有着较大诊断难度, 经常会出现 漏诊情况。因此,采用 PET-CT 技术加以明确。另外, 从本文研 究得知, MRI 诊断准确率高于 CT, MRI 诊断直径 $\leqslant 3.0 \mathrm{~cm}$ 的肿 瘤准确率高于增强 CT, 而直径 $>3.0 \mathrm{~cm}$ 者, 却无明显差异。由此 提示, 针对比较大的肝癌肿块, 采用 MRI、CT 都能得到好的诊 断准确率, 但对于直径 $\leqslant 3.0 \mathrm{~cm}$ 者, CT 对少血供于动脉期不强 化, 因而鉴别难度大。而对于 MRI, 其则可以较好地将早期成 像速度较慢的问题给克服掉, 提高场强, 因而可以达到提高小 肝癌检出率的目的。

综上,采用增强 CT、MRI 诊断原发性肝癌, MRI 诊断准确 率高于增强 CT, 特别是直径 $\leqslant 3 \mathrm{~cm}$ 的肿瘤, 其有着更高的准确 率。

\section{参考文献}

[1]赵年,李春华,李德秀,等.MRI 与 $\mathrm{CT}$ 多期动态增强扫描诊断肝 硬化伴肝癌的效果比较 [J].中国 CT 和 MRI 杂志,2017,15(5):85-87.

[2]郑义兰,崔惠勤.不同影像学手段在甲胎蛋白阴性肝癌术后复 发中的早期沴断价值[J].广西医科大学学报,2018,35(5):707-709.

[3] 邢卫红,冯爱东, 李若旭,等.CT 平扫及增强检查对肝硬化门脉高 压伴脾功能六进的应用价值[J].中国 CT 和 MRI 杂志,2017,15(1):79-82. 